\title{
Innovation in Phytotherapy: Is a New Regulation the Feasible Perspective in Europe?
}

Authors

Affiliations
Paola Minghetti ${ }^{1}$, Silvia Franzè ${ }^{1}$, Viviana Zaccara ${ }^{1}$, Floriana Raso ${ }^{2}$, Paolo Morazzoni ${ }^{3}$

${ }^{1}$ Dipartimento di Scienze Farmaceutiche, Facoltà di Scienze del Farmaco - Università degli Studi di Milano, Milano, Italy

${ }^{2}$ Loacker Remedia srl, Egna, Italy

${ }^{3}$ Indena S. p. A., Milano, Italy
Key words

- herbal medicinal products

- phytotherapy

- innovative herbal medicaments received Dec. 11, 2015

revised February 23, 2016

accepted March 2, 2016

\section{Bibliography}

Dol http://dx.doi.org/ 10.1055/s-0042-104509

Published online May 2, 2016

Planta Med 2016; 82: 591-595

(c) Georg Thieme Verlag KG

Stuttgart · New York .

ISSN 0032-0943

\section{Correspondence}

Prof.ssa Paola Minghetti

Docente di Tecnologia,

Socioeconomia e Legislazione Farmaceutiche

Direttore della Scuola di

Specializzazione in Farmacia

Ospedaliera

Dipartimento di Scienze

Farmaceutiche

Facoltà di Scienze del Farmaco -

Università degli Studi di Milano

Via G. Colombo 71

20133 Milano

Italy

Phone: + 390250324639

Fax: + 390250324657

paola.minghetti@unimi.it

\section{Abstract}

$\nabla$

Classical multicomponent preparations mostly derived from traditional usages in Western and Eastern phytotherapy have been under-evaluated for a long time as potential new pharmaceutical products. The regulatory scenario, in particular at the European level, has only recently considered these aspects proposing harmonized guidelines for the pharmaceutical registration of traditional herbal products. Nevertheless, a specific regulation for innovative products based on the combination of precious knowledge arising from traditional usages and modern scientific advancements is still missing. In this paper, we propose a critical review of the current situation with the specific aim of contributing to create a more favorable regulatory environment for the pharmaceutical registration of new and innovative herbal medicinal products.

\section{Introduction}

\section{$\nabla$}

Since the early history of medicine and up until the 20th century, therapeutic aids have been almost exclusively based on multicomponent products obtained from natural sources, including botanical derivatives as an important part. Only at the beginning of the 20th century, generations of Western medicine scientists, mostly inspired by Paul Ehrlich's "magic bullet" paradigm, started to spend all their efforts in finding new, powerful therapeutic compounds aimed at clearly defined molecular targets. This approach, mainly based on HTS applied to natural, semisynthetic, and total synthetic derivatives, has been developed for decades throughout the past 50 years and is mainly focused on the combination of the concepts of "maximal selectivity" and "maximal potency". This paradigmatic approach has represented an unchanged milestone in the context of

\section{Abbreviations}

$\nabla$

CTD: Common Technical Document

EMA: $\quad$ European Medicines Agency

FDA: $\quad$ Food and Drug Administration

HMPs: Herbal Medicinal Products

HMPCs: Committee on Herbal Medicinal Products

HTS: $\quad$ High-Throughput Screening

IND: Investigational New Drug

MA: $\quad$ Marketing Authorization

NBEs: New Biological Entities

NCEs: New Chemical Entities

THMPs: Traditional Herbal Medicinal Products WEU: Well-Established Use drug discovery and development in the last decades and has undoubtedly yielded successful drugs, like microtubule-targeting anticancer agents (taxol, taxotere, ixabepilon), selective proton-pump inhibitors (R-lansoprazole), and EGFRtyrosine kinase inhibitors (iressa). Even looking at this scenario of single new molecules introduced into the pharmaceutical market, it is worth to consider that natural sourcing played a major role in recent years as well. From 1983 to 1994, about $40 \%$ of novel drugs approved in North America were derived from natural compounds and approximately $70 \%$ of the new chemical entities reported between 1981 and mid-2006 arose from research on natural products [1].

In spite of this impressive effort, the rate of approval of new drugs, mainly discovered and developed following the approach described above, has declined markedly in the last 20 years. Main reasons were failure due to lack of efficacy in the 
Table 1 Comparison among possible drugs derived from plants and the modules of CTD required.

\begin{tabular}{llllll} 
& THMP & Well-established use & $\begin{array}{l}\text { Innovative- } \\
\text { (WEU) }\end{array}$ & $\begin{array}{l}\text { Copy of full dossier } \\
\text { drug }\end{array}$ & $\begin{array}{l}\text { THMP with some } \\
\text { innovation }\end{array}$ \\
\hline CTD & Simplified & Bibliographical & Complete & Simplified & Hybrid \\
\hline CTD: Module 3 (quality) & Full & Full & Full & Full & Full \\
\hline CTD: Module 4 (safety) & - & Full (Bibliographical) & Full & - & To evaluate case by case \\
\hline CTD: Module 5 (efficacy) & - & Full (Bibliographical) & Full & Bioequivalence study & To evaluate case by case
\end{tabular}

advanced phase of clinical testing and/or presence of important side effects [2].

In addition, evidence is steadily increasing that due to their complex etiopathogenesis, a number of relevant diseases require combination therapy, which may include several single-component drugs. Combination therapy is usually designed both to modulate multiple processes involved in the disease and also to reduce resistance, which is commonly induced by a single-agent treatment. Combination therapy plays a particularly important role in the treatment of infectious diseases such as HIV, tuberculosis, and malaria and complex chronic diseases like cancer, atherosclerosis, and metabolic syndrome. In this context, another concept gaining favor is that an approach strictly linked to HTS may only select interesting compounds with high activity, whose affinity to a target enzyme or receptor is strong but, on the contrary, it would be undoubtedly missing any prodrug and, more importantly, potential synergism among active principles in the same multicomponent product $[3,4]$.

All these issues are a matter of current discussion within the scientific community, which is wondering whether bioactive mixtures of natural products should be conceptually and concretely reconsidered for the development of new therapeutic approaches. This "rethinking" has also been fueled by the recent wave of reevaluation of Eastern traditional medical systems such as traditional Chinese medicine (TCM) and Indian Ayurveda, due to increasing popularity [5]. These approaches, indeed, are more oriented to consider natural product mixtures more appropriate to prevent and treat complex, pleiotropic diseases than single active pharmaceuticals. Nevertheless, even within Western traditional phytotherapy, the old concept of "phyto-complex" was strictly linked to the identification of the active principle as a multiplicity of molecules rather than a single component of the mixture. From this perspective, it has to be noted that many attempts to identify the single actually working active principle have failed [(i.e., Ginkgo biloba L. (Ginkgoaceae), Hypericum perforatum L. (Hypericaceae), Panax ginseng C.A.Mey. (Araliaceae), Valeriana officinalis L. (Caprifoliaceae)]. Taking into account that traditional medicine, which often includes complex botanical derivatives, is as an extremely rich source of potential innovation in the pharmaceutical area, a great expectation arises constantly in the scientific and pharmaceutical industry community, but in most cases such expectation is frustrated by the constraints of the regulatory systems. Actually, these are mainly oriented to the classical "magic bullet" approach and consequently prefer the time-consuming strategy of identifying the active principles of a complex mixture and further developing them according to the regular mainstream pharmaceutical rules. In the best cases, they accept a multicomponent product as a pharmaceutical entity, but impose a discouraging first-line safety and efficacy assessment, which most often hampers the development of the product. Moreover, an additional discouraging factor is the fact that in some cases the products can be put on the market as "food" (i.e., health food or food supplement) $[6,7]$ and marketed with claims sometimes "mimicking" the therapeutic effect, or as if they were medical devices with claims which are really superimposable to those of medicinal products.

The discussion on the potential of complex natural product mixtures is also positively influenced by the undeniable advancement in the field of available analytical technologies which, besides the currently used sensitive and selective variants of TLC, GC, and HPLC, can also include additional methodologies such as combined chromatography-mass spectrometry and even combined chromatography-NMR. When applied to multicomponent products, these techniques can really allow a detailed definition of their composition $[8,9]$. These approaches are also an effective tool to prepare lab-scale, pilot-scale and definitely industrialscale batches of rigorously reproducible standardized products satisfying all pharmaceutical grade requirements for preclinical/ clinical development and subsequent marketing authorization. Considering the points above, and based on the level reached by technological and scientific knowledge on HMPs, we really wonder whether a new approach can be considered at the European regulatory level in order to allow a simplified pharmaceutical development for innovative HMPs and therefore increase the number of safe and effective products on the market. In particular, our suggestion is to consider that a more favorable regulatory environment could help establish a faster approach to the clinical development of innovative HMPs aimed at improving the therapeutic index of traditionally utilized products, meaning in detail: - New standardization method focused on active principles recently proved to be pivotal for the effect.

- Removal of undesired compounds.

- Improvement of oral bioavaibility.

- Rational combinations with other HMPs for new therapeutic targets.

\section{Regulatory Aspects}

$\nabla$

As of today, in order to reach the market, according to European medicine legislation (Directive 2001/83/EC as amended by Directive 2004/24/EC), medicinal products containing herbal substances/preparations must fall within one of the following five categories ( Table 1):

1. A product can be a traditional herbal medicinal product if it has a traditional use and sufficient safety data and plausible efficacy. This product can be registered through a simplified registration procedure (article 16a) [10].

2. A medicinal product can be considered well established if, according to scientific literature, the applicant can demonstrate that the active substances have had a well-established medicinal use in EU for at least 10 years, and have a recognized efficacy and an acceptable level of safety. The product can be authorized through a full bibliographic CTD (article 10a). 
3. A product including new active substances can be marketed only after a competent authority's evaluation of safety and efficacy data resulting from the company development program. The product can be authorized through a full CTD (article 8).

4. A product can be considered a copy if it has the same qualitative and quantitative composition in active substances, the same pharmaceutical form, and bioequivalence (demonstrated by appropriate bioavailability) to the reference product. In this case, it can be authorized through a simplified CTD [article 10 (1)].

Historically, the regulatory approach to herbal substances and preparations has been controversial within the European countries, and several products classified differently as drugs, food and dietary supplements, cosmetics, and medical devices coexist on the market. Then, aiming to harmonize the regulation of such natural products, the European Parliament issued Directive 2004/24/EC, amending, with regard to THMPs, the Directive n. 2001/83/EC about a community code relating to herbal products presenting therapeutic claims [11]. According to this directive, a simplified pharmaceutical registration could be granted to herbal medicinal products with a history of traditional use in Europe. Considering this last change, two classes of HMPs are today legally recognized in Europe: WEU and THMPs. WEU are those HMPs with proven clinical efficacy and at least ten years use in the European Union. THMPs (introduced for the first time by Directive 2004/24/EC) do not need to provide clinical data in the registration dossier. Their features are the following:

- Traditional usage for at least 30 years, of which at least 15 is in the European Community.

- Oral, external, or inhaled use.

- Self-medication drugs: the intervention of a medical doctor should not be required for diagnosis, drug prescription, and therapy follow-up.

- Therapeutic indications are appropriate for THMPs.

- Administration at a specific concentration and dosage.

- Not harmful if properly used; plausible efficacy and pharmacological effects based on long-term experience and usage.

THMPs may be granted market authorization through simplified registration not requiring preclinical and clinical studies.

The application for a THMP registration must therefore be accompanied by all the required information for MA for a medicinal product, with the exception of clinical data and experimental preclinical data, and must include as well:

- Bibliographical evidence that the product has a traditional use (if an EMA monograph exists, it can be used as a certification for the traditional use for the submitted indications).

- Bibliographical and expert reports on the medicines' safety.

- Registration application or MA for the product obtained in an EU member state or other country, and all the information regarding any refusal received in an EU member state or other country, including the explanation of the decision leading to that outcome.

The primary objective of Directive 2004/24/EC was to ensure quality and safety of these products and clearly define therapeutic indications. For this purpose, an EMA HMPC has been established and is actively working on the elaboration of lists of herbal substances, vegetal preparations and combination products, and plant extract monographs. More than 150 monographs have already been published, all supported by a full bibliographic review on quality, safety, and efficacy for each plant extract. Despite this huge effort at the European level, the transposition of this legal intent is still pending in many European countries. The registra- tion of botanicals as drugs is not a centralized procedure and it is left to national agencies by means of a national or mutual recognition procedure [Council Regulation (EEC) No 2309/93]. Many agencies are still skeptical towards botanical drugs and the registration process is expensive and extremely slow, wasting a real market opportunity. Furthermore, the European Food Safety Authority (EFSA) suspended its opinion on botanical claims and criteria that should be adopted to avoid ambiguity and define clear links between food supplements and HMPs, which are still confused. The result is that still unregistered products, food supplements, and HMPs coexist in many markets, often with the same dosage and indications. Finally, an additional issue is represented by the increasing number of medical devices with therapeutic claims based on botanical active principles on the market. This is allowed if the main mechanism of action is physical (i.e., the formation of a polymeric film that acts to protect the mucosa). In this case, therapeutic effects can be accepted and the dossier to obtain the EC mark has to include some demonstration, even bibliographical, of clinical efficacy and safety.

In this context, it is worth considering that as long as this regulatory environment for the registration of HMPs remains unchanged, the majority of novel opportunities will remain in the food area or other parallel registration tracks and will therefore be left out of the pharmaceutical area, which indeed should be the most obvious for HMPs.

On the other hand, borderline situations are growing and multiplying in order to find market opportunities for innovative phytoactive principles that do not find their natural way in being acknowledged as medicinal products.

With this regard, a relatively recent hope for a potential opening to reconsider "natural multicomponent products" as proper pharmaceuticals and avoid the "classical" single-component approach is represented by the US "Botanical Drug Products-Guidance for industry". This Guidance was officially delivered in June 2004 by the FDA through the Center for Drug Evaluation and Research (CDER) and very recently updated [12]. The declared purpose of the Guidance is to consider botanical drugs' special features and through further adjustment during the FDA review process, to "facilitate" the development of new therapies from botanical sources. These therapies are intended for use in the diagnosis, cure, mitigation, treatment, or prevention of diseases in humans. It is also worthwhile to consider that the FDA Guidance has been proposed concomitantly to the existence of a dietary supplement market where all herbal preparations are included and which does not pose a pre-marketing approval. In this context, botanicals contained in dietary supplements already on the US market could theoretically be upgraded to pharmaceuticals according to the FDA Guidance.

According to the Guidance, a botanical drug can be defined as a product that contains vegetable materials as ingredients, including plant materials, algae, macroscopic fungi, or combinations thereof. It may be available as (but not limited to) a solution (e.g., tea), powder, tablet, capsule, elixir, topical, or injectable. Fermentation products, highly purified (or chemically modified) botanical substances, genetically modified plants, allergenic extracts, and vaccines containing botanical ingredients are not contemplated.

Based on the requirements defined by the Guidance, multicomponent botanical products with previous utilization both in the USA and/or other countries can be considered for initiating a proof of concept phase II clinical investigation, with a limited dossier. 
According to FDA guidelines for industry, the development path for botanical drug products consists of the following steps [13]:

- Discovery phase: screening, ethnobotany, bioinformatics.

- Plant acquisition, genotype selection, optimization.

- Product characterization, extraction method development, optimization, standardization.

- In vitro and in vivo efficacy validation.

- IND application to FDA.

- Clinical development (Phase 1 and Phase 2).

- Additional toxicology, mode of action, pharmacokinetics, drug interactions.

- Quality system (QS)/current good manufacturing practice (CGMP) protocols.

- Clinical development (Phase 3).

- New drug application (NDA) approval by FDA.

- Commercialization.

In particular, the CMC section does not include a "mandatory" request to identify the active components of the product, and therefore further purifications are not required. On the other hand, additional quality controls are requested for initial raw materials and the product to be tested in clinical studies. Preclinical investigation requirements are limited and even preclinical toxicology may be replaced by evidence of previous human use and can therefore be postponed to further development steps, usually within phase III. Theoretically, this simplified approach is significantly shortening the full length of the development of a botanical drug as compared to the "classical" pharmaceutical development of new molecular entities.

In fact, the Pharma way to develop NCEs/NBEs requires many years (approximately 15): from the first to the fifth year, the focus is the research target identification; from the fifth to the seventh year, the target is the discovery lead identification and optimization; and from the seventh to the fifteenth year, the target is the development at preclinical and clinical phases. Through the new approach is based on traditional experience, considering that much information is available, the registration is achieved in approximately eight years. The value of this difference is shortened research and discovery leveraging ancient knowledge and faster clinical development including earlier phase II trials as proofs of concept.

Like with other "innovative" changes, the newly introduced Guidance has also encountered a relatively slow grade of acceptance by the pharmaceutical environment. As of today, only two products have been finally registered under its umbrella: Veregen ${ }^{\circledR}$ for the treatment of genital warts (2006) and, more recently, Fulizaq ${ }^{\circledR}$ for the treatment of HIV-associated diarrhea (2012). Nevertheless, an increasing number of INDs have been submitted in the last years, showing that the level of acceptance of this new opportunity is gradually growing.

In addition, recent agreements between big multinational companies and smaller companies with a portfolio of traditionally used multicomponent botanical products have been established with the specific aim of developing new botanical drugs, taking advantage of the Guidance.

Finally, it is also worth to note that Chinese and Indian governments, and more recently also South Korean authorities, are strongly suggesting that local companies consider the US Guidance as a potential channel for "modernizing" traditional Chinese medicine, Indian Ayurveda, and Korean traditional medicine, respectively, considering the possibility to validate old traditional formulas mostly based on multicomponent products in a Western country. As a concrete result of this policy, an impressive number of INDs have been submitted to the FDA by Chinese, Indian, and South Korean companies, sometimes through American subsidiaries.

\section{Closing Remarks and Authors' Proposal}

Looking at this rapidly evolving scenario with the eyes of European companies committed to pharmaceutical innovation, it is like feeling a "lost opportunity". At the present time, the regulatory European scenario for phytotherapy is wasting efforts in the difficult task of properly registering and harmonizing the old medicinal products while scarcely considering the traditional enormous portfolio of commonly utilized products as a potential "reservoir" of innovation when they are not addressed according to the "magic bullet" paradigm [14]. In fact, the two existing directives [Traditional Herbal Medicine (article 16a, Directive 2004/24/EC) and Well Established Use (art 10a, Directive 2001/ $83 / \mathrm{EC})$ ], by definition, are both aimed at recognizing the option of a "simplified" pharmaceutical registration only if the products have proven long-term usage, in the first case, or based on evidence from previous randomized controlled clinical trials, in the second case. This should be seen as a first step to harmonize the market and properly register HMPs. The following step should be the "simplified" option in order to create innovation in the field of phytotherapy by starting to consider traditionally used multicomponent products or their improved formulations as new botanical drugs to be developed. In fact, the THMP legislation limits the registration as medicinal products to herbal compounds with the indications approved by the HMPC and reported in the monograph (for mild, self-medication pathologic conditions and short-period treatment). To facilitate the MA of HMPs with some innovations, the requirement of a full dossier seems to be not needed in terms of safety and efficacy guarantee, and registration can be quickened using a hybrid application. According to article 10 (3) of the Directive 2001/83/EC, a medicinal product having new active substances, new therapeutic indications, strength, pharmaceutical form, or route of administration with respect to a reference medicinal product can be authorized with a hybrid application, providing some new data from preclinical and clinical studies. This legal basis may be considered applicable also to herbal preparations as long as the WEU is assumed as a reference medicinal product. Instead, the THMP does not fall into the strict definition of a reference medicinal product given by the EMA, since a full dossier is missing ( $\odot$ Table 1 ). Updated guidelines allowing a hybrid recording for THMPs with a certain grade of innovation in terms of manufacturing or therapeutic indication would increase the grade of acceptance of this trend. These guidelines would define a possible simplification to be granted in terms of characterization, preclinical studies, and clinical trials. For example, while completely new plants have to be characterized in terms of safety with the requirement of a full preclinical toxicological evaluation, plants for which there is evidence of use should benefit from a simplified access to phase II, even when proposed for new indications.

In this context, we suggest European authorities consider this potential opportunity and evaluate a more flexible approach for the future in order to help have more innovative therapeutic options available based on the huge portfolio of traditional remedies belonging to our phytotherapy armamentarium without forgetting the legacy from extra-European medical approaches when a large amount of safety data is available. 
Moreover, it is clear that a speed-up of the registration of THMPs and WEU herbal derivatives with some grade of innovation would allow an appropriate environment to create new and innovative therapeutic uses for HMPs.

\section{Conflict of Interest}

\section{$\nabla$}

The authors Paola Minghetti, Silvia Franzè, and Viviana Zaccara declare no conflicts of interest. Paolo Morazzoni is a Scientific Director at Indena S.p.A. Floriana Raso is a Regulatory Affairs Director at Loacker Remedia srl.

\section{References}

1 Newman DJ, Cragg GM. Natural products as sources of new drugs over the last 25 years. J Nat Prod 2007; 70: 461-477

2 Morrow JK, Tian L, Zhang S. Molecular networks in drug discovery. Crit Rev Biomed Eng 2010; 38: 143-156

3 Gertsch J. Botanical drugs, synergy and network pharmacology: forth and back to intelligent mixtures. Planta Med 2011; 77: 1086-1098

4 Schmidt B, Ribnicky DM, Poulev A, Logendra S, Cefalu WT, Raskin I. A natural history of botanical therapeutics. Metabolism 2008; 57: S3-S9

5 Ramawat KG. Herbal drugs: ethnomedicine to modern medicine. Berlin, Heidelberg: Springer-Verlag; 2009

6 Silano V, Coppens P, Larrañaga-Guetaria A, Minghetti P, Roth-Ehrang $R$. Regulations applicable to plant food supplements and related products in the European Union. Food Funct 2011; 2: 710-719
7 Bilia AR. Herbal medicinal products versus botanical-food supplements in the European market: state of art and perspectives. Nat Prod Commun 2015; 10: 125-131

8 Wolfender JL, Marti G, Thomas A, Bertrand S. Current approaches and challenges for the metabolite profiling of complex natural extracts. J Chromatogr A 2015; 1382: 136-164

9 Bilia AR. Science meets regulation. J Ethnopharmacol 2014; 158: 487494

10 European Medicines Agency. Guideline on the use of the CTD format in the preparation of a registration application for traditional herbal medicinal products. EMA/HMPC/71049/2007, Rev. 2; 2015. Available at http://www.ema.europa.eu/docs/en_GB/document_library/Regulatory_and_procedural_guideline/2015/04/WC500185353.pdf. Accessed February 22, 2016

11 Knöss W, Chinou I. Regulation of medicinal plants for public health European community monographs on herbal substances. Planta Med 2012; 78: 1311-1316

12 U.S. Department of Health and Human Services, Food and Drug Administration, Center for Drug Evaluation and Research (CDER). Botanical Drug Development Guidance for Industry, Revision 1: August 2015 (Pharmaceutical Quality/CMC). Available at http://www.fda.gov/downloads/ Drugs/GuidanceComplianceRegulatoryInformation/Guidances/ UCM458484.pdf. Accessed February 22, 2016

13 Schmidt BM, Ribnicky DM, Lipsky E, Raskin I. Revisiting the ancient concept of botanical therapeutics. Nat Chem Biol 2007; 3: 360-366

14 Fürst $R$, Zündorf I. Evidence-based phytotherapy in Europe: where do we stand? Planta Med 2015; 81: 962-967 